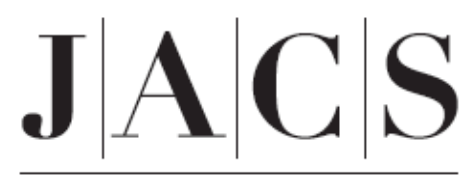

A R T I C L E S

\title{
Operating Molecular Elevators
}

Jovica D. Badjic, ${ }^{\dagger}$ Célia M. Ronconi, ${ }^{\dagger}{ }^{J}$. Fraser Stoddart, ${ }^{* \dagger}$ Vincenzo

Balzani, ${ }^{\ddagger}$ Serena Silvi, ${ }^{\ddagger}$ and Alberto Credi ${ }^{*, \neq}$

Contribution from the California NanoSystems Institute and Department of

Chemistry and Biochemistry, University of California, Los Angeles, 405 Hilgard

Avenue, Los Angeles, California 90095-1569, and Dipartimento di Chimica

“Giacomo Ciamician”, Università di Bologna, Via Selmi 2, I-40126 Bologna, Italy.

E-mail: alberto.credi@unibo.it; stoddart@chem.ucla.edu

\section{Supporting Information}

\footnotetext{
University of California, Los Angeles.

* Università di Bologna.
} 
Synthesis. For the synthesis (Scheme $\mathrm{S} 1$ ) of the $\left[6 \mathrm{H}_{3}\right]\left[\mathrm{PF}_{6}\right]_{9}, 1,3,5$-tris ( $p$-formyl phenyl)benzene was condensed with (4-aminomethyl)benzyl alcohol and the imine reduced $\left(\mathrm{NaBH}_{4} / \mathrm{MeOH}\right)$ to the trisamine $\mathbf{9}$, which was Boc-protected to yield the triol $\mathbf{1 0}$ that was converted to the trischloride 11, followed by TFA deprotection in $\mathrm{CHCl}_{3}$ and counterion exchange $\left(\mathrm{NH}_{4} \mathrm{PF}_{6} / \mathrm{MeOH} / \mathrm{H}_{2} \mathrm{O}\right)$ to afford $\left[\mathbf{1 2}_{3}\right]\left[\mathrm{PF}_{6}\right]_{6}$.

Scheme S1. The synthesis of the trifurcated guest salt $\left[4 \mathrm{H}_{3}\right]\left[\mathrm{PF}_{6}\right]_{6}$ that was, at elevated temperature $\left(75^{\circ} \mathrm{C}\right)$, converted into the mechanically interlocked bundles - namely the molecular elevators $\left[5 \mathrm{H}_{3}\right]\left[\mathrm{PF}_{6}\right]_{9}$ and $\left[8 \mathrm{H}_{3}\right]\left[\mathrm{PF}_{6}\right]_{9}-$ using the tris-crown ethers 2 and 7 , respectively
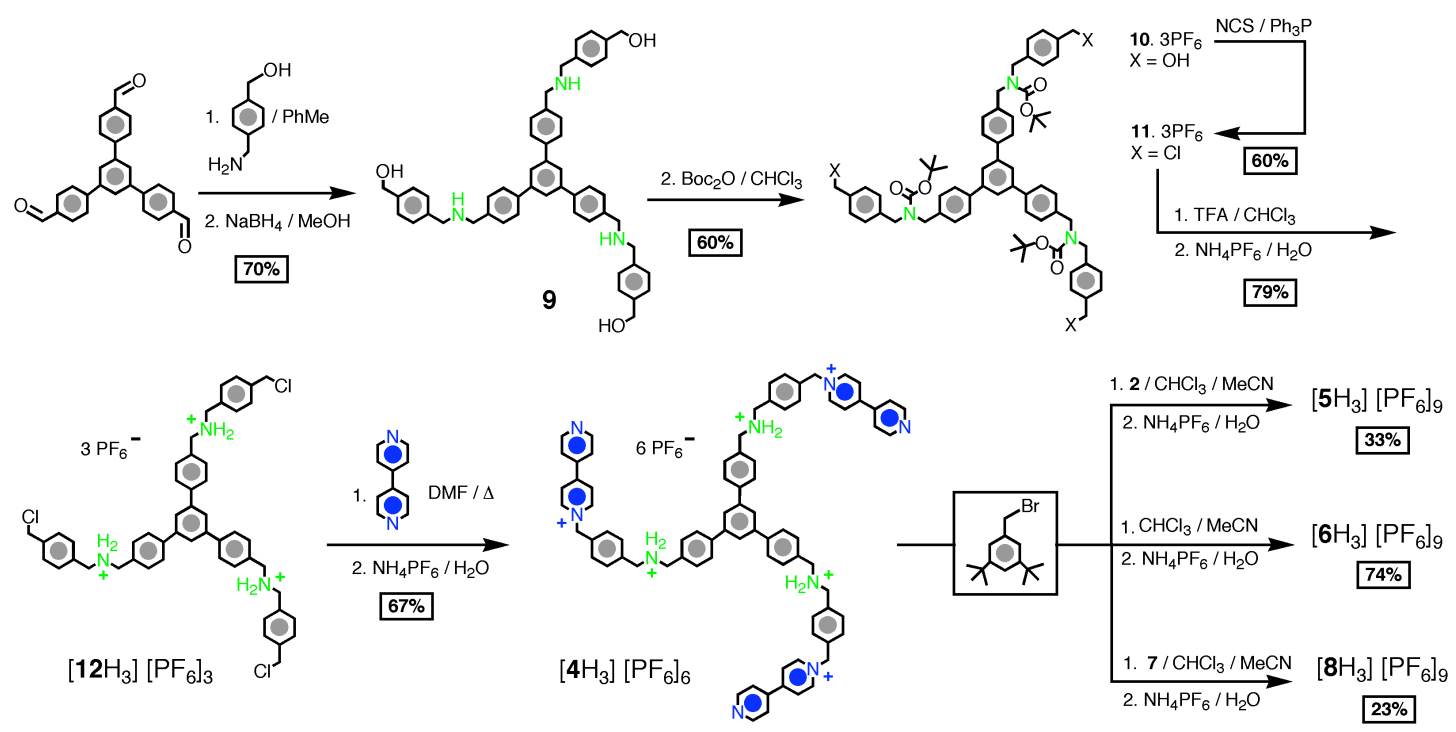

The precursor to the trifurcated rig $\left[\mathbf{4 H}_{3}\right]\left[\mathrm{PF}_{6}\right]_{6}$ was prepared by monoalkylation of 4,4'-bipyridine with the trischoride. This precursor compound was converted at elevated temperature $\left(75{ }^{\circ} \mathrm{C}\right)$ to the molecular elevators $\left[5 \mathrm{H}_{3}\right]\left[\mathrm{PF}_{6}\right]_{9}$ and $\left[8 \mathrm{H}_{3}\right]\left[\mathrm{PF}_{6}\right]_{9}$ utilizing a 
threading-followed-by-stoppering approach by complexing them with the tris-crown ether 2 and tris-crown ether 7, respectively, and then alkylating the terminal bipyridine units with (1) 3,5-di-tert-butylbenzylbromide, followed by (2) counterion exchange $\left(\mathrm{NH}_{4} \mathrm{PF}_{6} / \mathrm{MeOH} / \mathrm{H}_{2} \mathrm{O}\right)$.

Scheme S2. The synthesis of the tris-crown ether 7

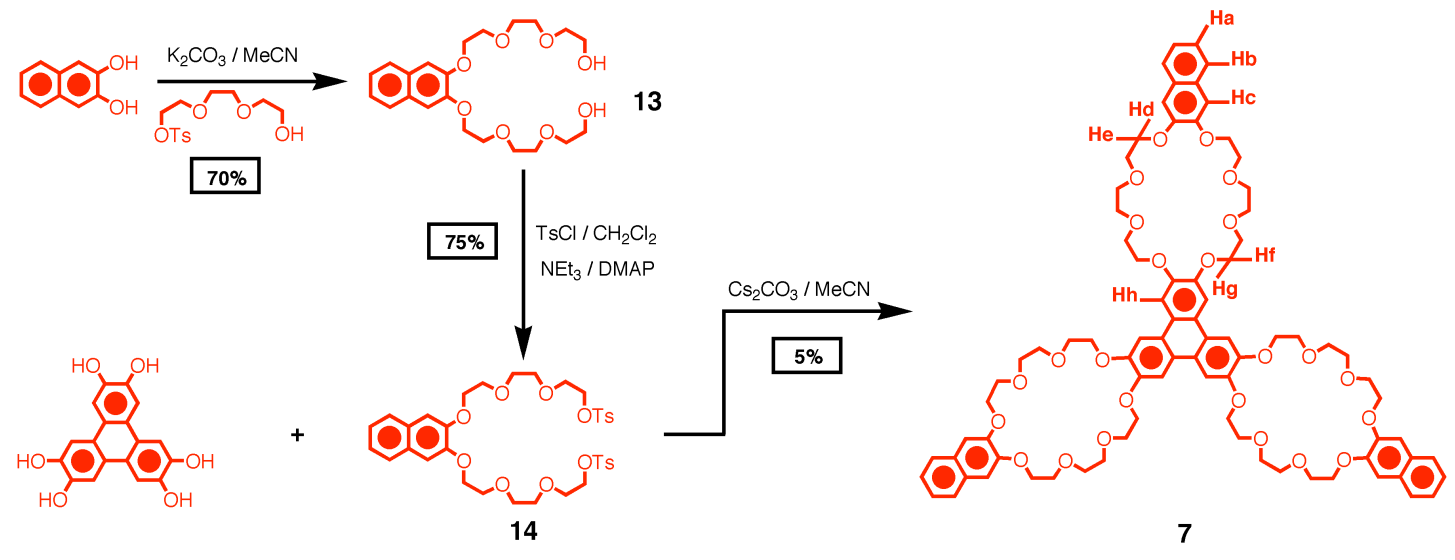

The synthesis of the tris-crown ether 2 has already been reported. ${ }^{\mathrm{S} 1}$ The tris-crown ether 7 was obtained (Scheme S2) in 5\% yield following a triple macrocyclization $\left(\mathrm{Cs}_{2} \mathrm{CO}_{3} / \mathrm{DMF}\right)$ between 2,3,6,7,10,11-hexahydroxytriphenylene (HHTP) and the appropriated bistosylate 14. HHTP was prepared according to literature procedures. ${ }^{\text {S2 }}$ The bistosylate 14 was obtained in two steps from 2,3-dihydroxynaphthalene, namely its bisalkylation $\left(\mathrm{K}_{2} \mathrm{CO}_{3} / \mathrm{MeCN}\right)$ with 2-[2-(2-p-tolylsulfonyloxy)ethoxy] ethanol to yield a diol 13 which, upon tosylation $\left(\mathrm{TsCl} / \mathrm{CH}_{2} \mathrm{Cl}_{2}\right)$, gave the desired product 14 .

Absorption and Luminescence Spectra. Table S1 and Figures S1, S2. 
Table S1. Absorption and Luminescence Data for the Molecular Elevators, their Molecular Components, and Model Compounds (MeCN, 298 K)

\begin{tabular}{|c|c|c|c|c|}
\hline \multirow[t]{2}{*}{ Compound } & \multicolumn{2}{|c|}{ Absorption } & \multicolumn{2}{|c|}{ Fluorescence $^{a}$} \\
\hline & $\lambda_{\max }(\mathrm{nm})$ & $\varepsilon\left(\mathrm{L} \mathrm{mol}^{-1} \mathrm{~cm}^{-1}\right)$ & $\lambda_{\max }(\mathrm{nm})$ & $\tau(\mathrm{ns})$ \\
\hline \multirow[t]{2}{*}{ 1,2-Dimethoxybenzene } & 227 & 13000 & \multirow[t]{2}{*}{310} & \multirow[t]{2}{*}{1.7} \\
\hline & 276 & 4300 & & \\
\hline \multirow[t]{3}{*}{ 1,2-Dimethoxynaphthalene } & 232 & 80000 & \multirow[t]{3}{*}{$326^{b, c}$} & \multirow[t]{3}{*}{8.3} \\
\hline & 267 & 5700 & & \\
\hline & 322 & 4700 & & \\
\hline $2,3,6,7,10,11-$ & $276^{b, d}$ & 100000 & $381^{b, e}$ & 9.5 \\
\hline Hexamethoxytriphenylene & 308 & 19000 & & \\
\hline \multirow[t]{2}{*}{2} & $277^{b, d}$ & 114000 & \multirow[t]{2}{*}{$383^{b, e}$} & \multirow[t]{2}{*}{9.0} \\
\hline & 307 & 23000 & & \\
\hline \multirow[t]{4}{*}{7} & 234 & 258000 & \multirow[t]{4}{*}{$384^{b, e}$} & \multirow[t]{4}{*}{8.7} \\
\hline & $279^{b, d}$ & 146000 & & \\
\hline & 310 & 36000 & & \\
\hline & 324 & 32000 & & \\
\hline$\left[6 \mathrm{H}_{3}\right]^{9+}$ & 261 & 151000 & 361 & 11.5 \\
\hline$\left[5 \mathrm{H}_{3}\right]^{9+}$ & 267 & 209000 & 370 & 10 \\
\hline \multirow[t]{3}{*}[8\mathrm{H}_{3}]{$^{9+}$} & 233 & 300000 & \multirow[t]{3}{*}{384} & \multirow[t]{3}{*}{7.9} \\
\hline & 267 & 216000 & & \\
\hline & 322 & 35000 & & \\
\hline
\end{tabular}

${ }^{a}$ Excitation was performed in the lowest energy absorption band. ${ }^{b}$ Structured band. ${ }^{c}$ Highest energy feature. ${ }^{d}$ Lowest energy feature. ${ }^{e}$ Highest intensity feature 


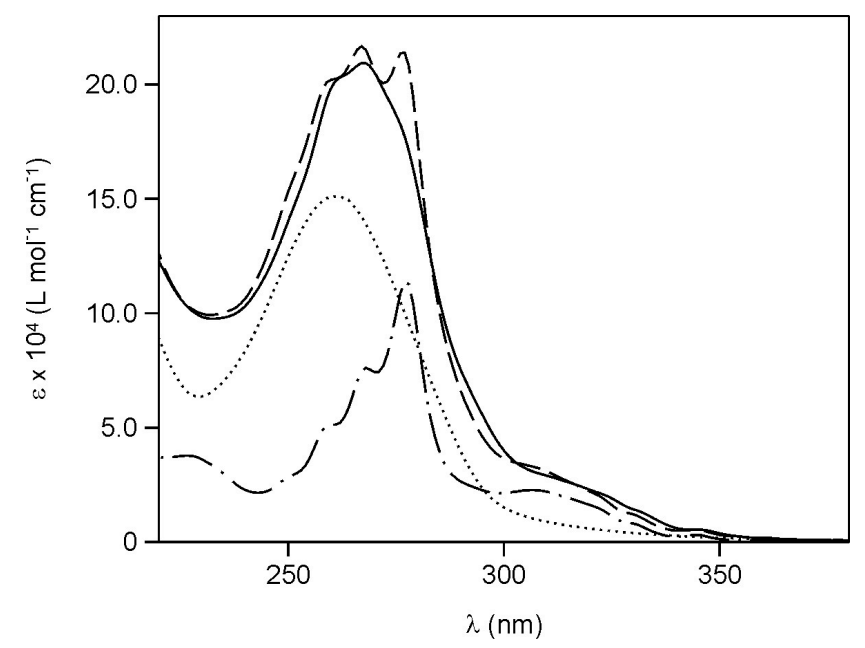

Figure S1. Absorption spectra $\left(\mathrm{MeCN}, 298 \mathrm{~K}\right.$ ) of the molecular elevator $\left[\mathbf{5 H}_{3}\right]^{9+}$ (full line) and of its molecular components, 2 (dashed-dotted line) and $\left[6 \mathrm{H}_{3}\right]^{9+}$ (dotted line). The sum of the spectra of the separated molecular components (dashed line) is also reported for comparison.

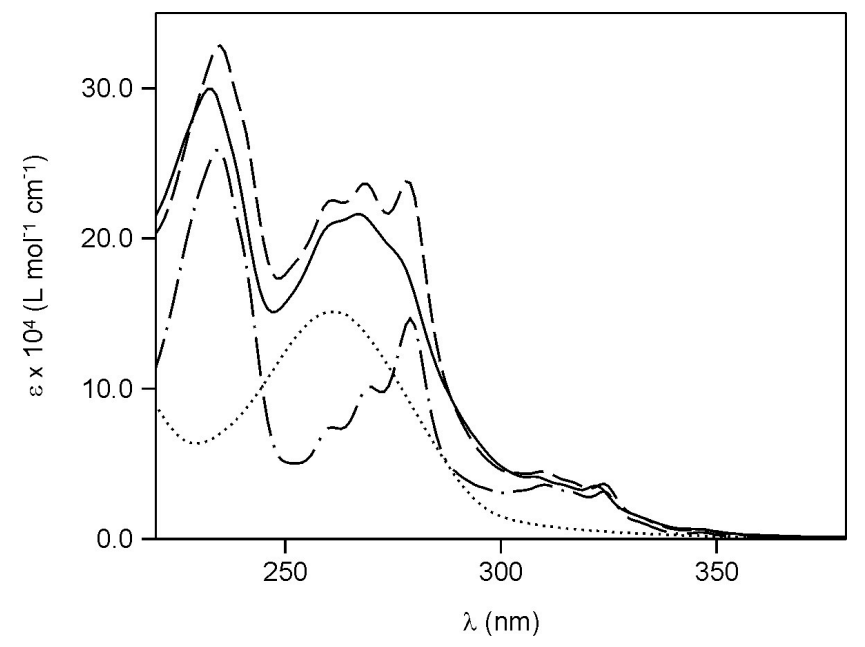

Figure S2. Absorption spectra $\left(\mathrm{MeCN}, 298 \mathrm{~K}\right.$ ) of the molecular elevator $\left[\mathbf{8 H}_{3}\right]^{9+}$ (full line) and of its molecular components, 7 (dashed-dotted line) and $\left[6 \mathrm{H}_{3}\right]^{9+}$ (dotted line). The sum of the spectra of the separated molecular components (dashed line) is also reported for comparison. 
Chemical Switching. Figures S3-S5.

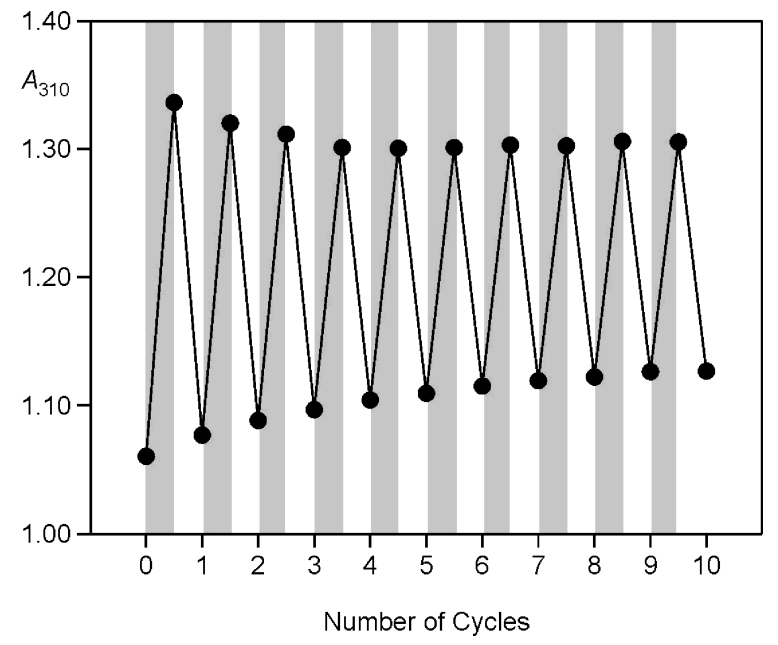

Figure S3. Changes in absorbance observed at $310 \mathrm{~nm}$ for a $7.1 \times 10^{-6} \mathrm{~mol} \mathrm{~L}^{-1}$ solution of $\left[5 \mathrm{H}_{3}\right]^{9+}$ on successive addition of stoichiometric amounts of base (dark areas) and acid (light areas). Conditions: $\mathrm{MeCN}, 298 \mathrm{~K}$, path length $=4.0 \mathrm{~cm}$.

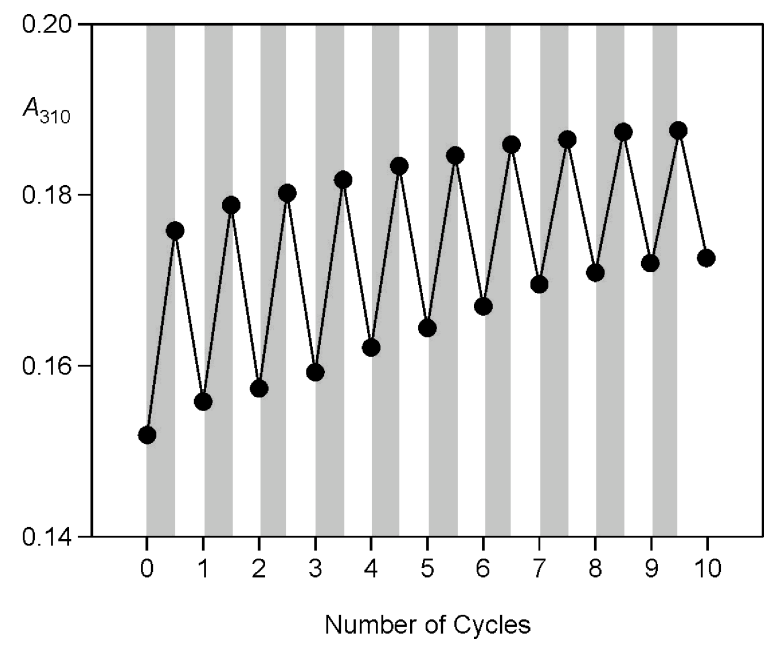

Figure S4. Changes in absorbance observed at $310 \mathrm{~nm}$ for a $4.0 \times 10^{-6} \mathrm{~mol} \mathrm{~L}^{-1}$ solution of $\left[8 \mathrm{H}_{3}\right]^{9+}$ on successive addition of stoichiometric amounts of base (dark areas) and acid (light areas). Conditions: $\mathrm{MeCN}, 298 \mathrm{~K}$, path length $=1.0 \mathrm{~cm}$. 


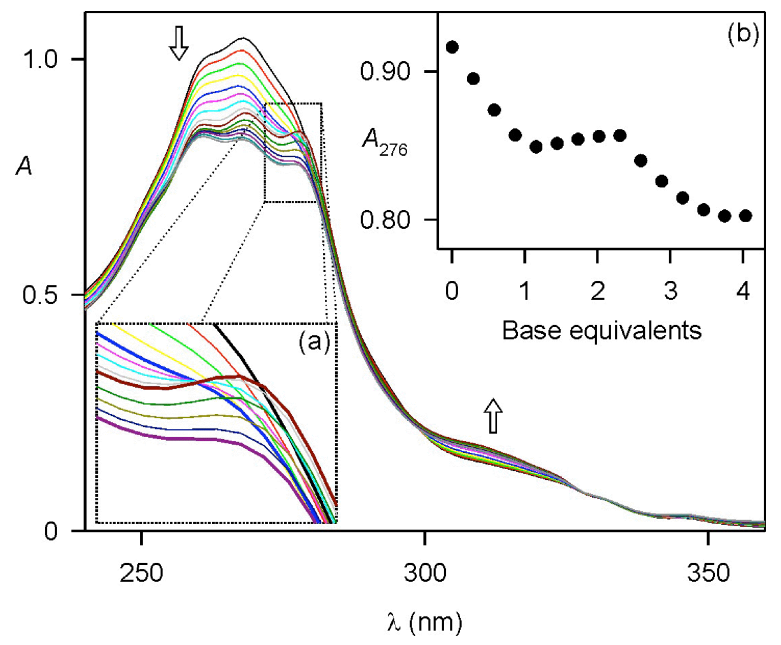

Figure S5. Absorption spectral changes observed upon titration at $298 \mathrm{~K}$ of a $5.0 \times 10^{-6}$ mol L ${ }^{-1} \mathrm{MeCN}$ solution of the molecular elevator $\left[5 \mathrm{H}_{3}\right]^{9+}$ with the $\mathrm{P}_{1}-t$-Bu base. Insets (a) and (b) show a magnification of the $272-282 \mathrm{~nm}$ region and the titration curve obtained from the absorbance changes at $276 \mathrm{~nm}$, respectively.

Molecular Modeling. Figure S6.

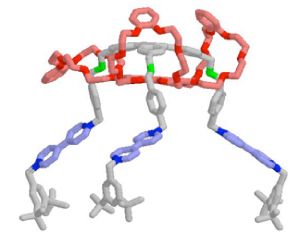

$\left[5 \mathrm{H}_{3}\right]^{9+}$

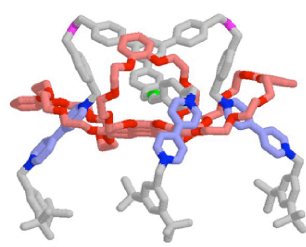

$\left[5 \mathrm{H}^{7}\right.$

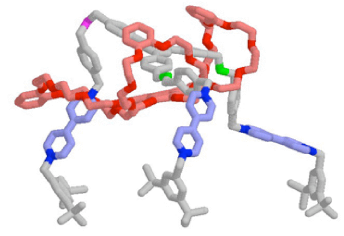

$\left[5 \mathrm{H}_{2}\right]^{8+}$

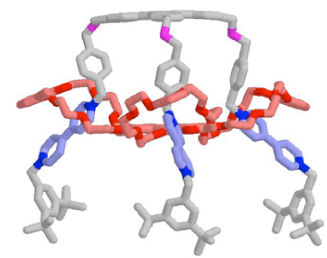

$5^{6+}$

Figure S6. Structures of the molecular elevator $\left[\mathbf{5 H}_{3}\right]^{9+}$ and of its deprotonated forms $\left[5 \mathrm{H}_{2}\right]^{8+},[\mathbf{5 H}]^{7+}$ and $\mathbf{5}^{6+}$, obtained by molecular mechanics calculations. 


\section{Experimental Section}

Material and Methods. All chemicals were purchased from Aldrich and were used without further purification. Solvents were purchased from Aldrich and purified according to literature procedures. Thin-layer chromatography (TLC) was performed on aluminum sheets coated with silica gel 60F (Merck 5554). The plates were inspected by UV light and, if required, developed in $\mathrm{I}_{2}$ vapor. Column chromatography was performed on silica gel 60 (Merck $40-60 \mathrm{~nm}, 230-400 \mathrm{mesh}$ ). Melting points were determined on an Electrothermal 9200 apparatus and reported uncorrected. ${ }^{1} \mathrm{H}$ and ${ }^{13} \mathrm{C}$ NMR spectra were recorded on either (i) a Bruker $600 \mathrm{MHz}$ (600 and $150 \mathrm{MHz}$, respectively) or (ii) Bruker Avance500 (500 and $125 \mathrm{MHz}$, respectively), using residual solvents as the internal standard. Samples were prepared using $\mathrm{CDCl}_{3}, \mathrm{CD}_{3} \mathrm{SOCD}_{3}$ and $\mathrm{CD}_{3} \mathrm{CN}$ purchased from Cambridge Isotope Laboratories. The chemical shift values were expressed as $\delta$ values, and the coupling constants values $(J)$ are in Hertz. The following abbreviations were used for signal multiplicities: $\mathrm{s}$, singlet; $\mathrm{d}$ doublet; $\mathrm{t}$ triplet; $\mathrm{m}$ multiplet. Fast atom bombardment mass spectrometry (FAB-MS) was performed on a VG ZAB-SE mass spectrometer, equipped with a krypton primary atom beam using a 3nitrobenzyl alcohol matrix. Cesium iodide or poly(ethylene)glycol were employed as reference compounds. Electron impact mass spectra (EI-MS) were obtained from a VG Prospec mass spectrometer. Matrix-assisted laser desorption ionization mass spectra (MALDI-MS) were recorded on a PerSeptives Biosystems instrument.

9. A mixture of 4-aminomethylbenzyl alcohol $(3.2 \mathrm{~g}, 23.3 \mathrm{mmol})$ and 1,3,5-tris $(p$ formylphenyl)benzene $(3.0 \mathrm{~g}, 7.7 \mathrm{mmol})$ was heated in PhMe $(100 \mathrm{~mL})$ under reflux 
overnight, while $\mathrm{H}_{2} \mathrm{O}$ was removed using a Dean-Stark trap. The reaction mixture was cooled down in a freezer to $-10{ }^{\circ} \mathrm{C}$ and the resulting suspension filtered to give the $1,3,5$ tris[(4-benzylalcoholiminomethyl)phenyl)]benzene as a white solid (4.02 g, $74 \%)$. Mp $166-168{ }^{\circ} \mathrm{C} ;{ }^{1} \mathrm{H}$ NMR $\left(500 \mathrm{MHz}, \mathrm{CD}_{3} \mathrm{SOCD}_{3}\right) \delta 4.50(\mathrm{~d}, J=4.5 \mathrm{~Hz}, 6 \mathrm{H}), 4.81(\mathrm{~s}, 6 \mathrm{H})$, 5.15-5.2 (br, 3H), $7.32(\mathrm{~s}, 12 \mathrm{H}), 7.92(\mathrm{~d}, J=8.0 \mathrm{~Hz}, 6 \mathrm{H}), 8.00-8.07$ (m, 9H), $8.58(\mathrm{~s}$, $3 \mathrm{H}) ;{ }^{13} \mathrm{C} \mathrm{NMR}\left(125 \mathrm{MHz}, \mathrm{CDCl}_{3}\right): \delta 64.7,65.0,125.4,127.1,127.4,128.1,128.8,135.4$, 138.6, 139.6, 141.7, 143.0, 161.5; HRMALDI-MS $m / z$ calcd for $\mathrm{C}_{51} \mathrm{H}_{46} \mathrm{~N}_{3} \mathrm{O}_{3} 748.3534$ $[\mathrm{MH}]^{+}$, found $748.3578 . \mathrm{NaBH}_{4}(1.02 \mathrm{~g}, 26.8 \mathrm{mmol})$ was added portionwise to a solution of this white solid in dry MeOH $(100 \mathrm{~mL})$ and dry THF $(100 \mathrm{~mL})$. After the reaction had been left to stir overnight, the solvent was removed in vacuum. The resulting solid was partitioned between THF $(150 \mathrm{~mL})$ and brine $(100 \mathrm{~mL})$. The aqueous phase was washed with THF $(100 \mathrm{~mL})$. The organic phases were combined, dried $\left(\mathrm{MgSO}_{4}\right)$, and the solvent removed under vacuum to give 9 as a yellow liquid $(3.6 \mathrm{~g}, 90 \%)$. ${ }^{1} \mathrm{H}$ NMR (500 MHz, $\left.\mathrm{CDCl}_{3}\right) \delta 3.74(\mathrm{~s}, 6 \mathrm{H}), 3.77(\mathrm{~s}, 6 \mathrm{H}), 4.50(\mathrm{~s}, 6 \mathrm{H}), 7.22(\mathrm{~d}, J=8 \mathrm{~Hz}, 6 \mathrm{H}), 7.43(\mathrm{~d}, J=8$ $\mathrm{Hz}, 6 \mathrm{H}), 7.67(\mathrm{~d}, J=8 \mathrm{~Hz}, 6 \mathrm{H}), 7.80(\mathrm{~s}, 3 \mathrm{H}) ;{ }^{13} \mathrm{C} \mathrm{NMR}\left(125 \mathrm{MHz}, \mathrm{CDCl}_{3}\right) \delta 50.7,50.9$, $61.9,123.3,124.2,124.9,125.7,126.5,129.1,137.6,138.6,139.4,140.2$; HRMALDI-MS $m / z$ calcd for $\mathrm{C}_{51} \mathrm{H}_{52} \mathrm{~N}_{3} \mathrm{O}_{3}, 754.4003\left[M+\mathrm{H}^{+}\right]$, found 754.3147.

10.3PF . The trisamine $9(2.7 \mathrm{~g}, 3.6 \mathrm{mmol})$ was dissolved in $\mathrm{CHCl}_{3}(450 \mathrm{~mL})$ and then di-tert-butyl dicarbonate $(7.9 \mathrm{~g}, 36.1 \mathrm{mmol})$ was added. The reaction mixture was left to stir for $24 \mathrm{~h}$, before being washed with aqueous $2 \mathrm{M} \mathrm{HCl}$ solution ( 2 x $200 \mathrm{~mL}$ ), and $\mathrm{H}_{2} \mathrm{O}$ (200 mL). The organic phase was dried $\left(\mathrm{MgSO}_{4}\right)$ and evaporated under reduced pressure to yield a crude product that was subsequently purified by column chromatography $\left(\mathrm{SiO}_{2}\right.$, $\left.\mathrm{CH}_{2} \mathrm{Cl}_{2}: \mathrm{MeOH}, 95: 5\right)$ to yield the title compound $10.3 \mathrm{PF}_{6}(2.3 \mathrm{~g}, 60 \%) . \mathrm{Mp} 68-70{ }^{\circ} \mathrm{C} ;{ }^{1} \mathrm{H}$ 
NMR (500 MHz, $\left.\mathrm{CDCl}_{3}\right) \delta 1.52(\mathrm{~s}, 27 \mathrm{H}), 4.36-4.51(\mathrm{br}, 12 \mathrm{H}), 4.71(\mathrm{~s}, 6 \mathrm{H}), 7.20-7.34$ (m, 6H), $7.35(\mathrm{~d}, J=8 \mathrm{~Hz}, 6 \mathrm{H}), 7.66(\mathrm{~d}, J=8 \mathrm{~Hz}, 6 \mathrm{H}), 7.77(\mathrm{~s}, 3 \mathrm{H}) ;{ }^{13} \mathrm{C}$ NMR $(125$ $\left.\mathrm{MHz}, \mathrm{CDCl}_{3}\right) \delta 28.4,48.7,48.9,65.0,80.2,124.8,127.2,127.4,127.6,127.9,128.2$, 128.4, 137.2, 139.9, 141.9, 155.9; HRMALDI-MS $\mathrm{m} / z$ calcd for $\mathrm{C}_{66} \mathrm{H}_{75} \mathrm{~N}_{3} \mathrm{O}_{9} \mathrm{Na}$, $1076.5401[M+\mathrm{Na}]^{+}$, found 1076.5425 .

11.3PF $_{6}$. Triphenylphosphine $(4.1 \mathrm{~g}, 15.8 \mathrm{mmol})$ in THF $(100 \mathrm{~mL})$ was added to $N$ chlorosuccinimide $(2.4 \mathrm{~g}, 18.1 \mathrm{mmol})$ in THF $(100 \mathrm{~mL})$ under an argon atmosphere, forming a white suspension. The triol $10(2.0 \mathrm{~g}, 1.9 \mathrm{mmol})$ dissolved in THF (40 mL) was then slowly added and the reaction mixture was left to stir overnight. The solvent was then evaporated off and the product purified by column chromatography $\left(\mathrm{SiO}_{2}\right.$, hexanes:EtOAc, 7:3). The product $11.3 \mathrm{PF}_{6}$ was obtained as an off-white solid (2.0 g, $95 \%)$. Mp 62-64 ${ }^{\circ} \mathrm{C} ;{ }^{1} \mathrm{H}$ NMR (500 MHz, $\left.\mathrm{CDCl}_{3}\right) \delta 1.57$ (s, 27H), 4.45 (s, 6H), $4.52(\mathrm{~s}$, $6 \mathrm{H}), 4.65(\mathrm{~s}, 6 \mathrm{H}), 7.22-7.40(\mathrm{~m}, 12 \mathrm{H}), 7.42(\mathrm{~d}, J=8 \mathrm{~Hz}, 6 \mathrm{H}), 7.71(\mathrm{~d}, J=8 \mathrm{~Hz}, 6 \mathrm{H})$, $7.82(\mathrm{~s}, 3 \mathrm{H}) ;{ }^{13} \mathrm{C} \mathrm{NMR}\left(125 \mathrm{MHz}, \mathrm{CDCl}_{3}\right) \delta 28.5,46.0,48.9,49.2,80.3,125.0,127.5$, $127.8,128.5,128.9,136.6,137.4,138.4,140.1,142.0,156.0 ;$ HRMALDI-MS $m / z$ calcd for $\mathrm{C}_{66} \mathrm{H}_{72} \mathrm{~N}_{3} \mathrm{O}_{6} \mathrm{Cl}_{3} \mathrm{Na}, 1130.4384[M+\mathrm{Na}]^{+}$, found 1130.4398 .

$\left[\mathbf{1 2 H}_{3}\right]\left[\mathbf{P F}_{6}\right]_{3}$. Trifluoroacetic acid $(20 \mathrm{~mL})$ was added to the tert-Boc-protected amine 11. $3 \mathrm{PF}_{6}(0.9 \mathrm{~g}, 0.8 \mathrm{mmol})$ in $\mathrm{CHCl}_{3}(120 \mathrm{~mL})$, and the reaction mixture was stirred at the room temperature for $16 \mathrm{~h}$. The solvent was evaporated under vacuum, and the residue dissolved in $\mathrm{MeOH}: \mathrm{Me}_{2} \mathrm{CO}$ (15 mL, 1:1). A saturated aqueous solution of $\mathrm{NH}_{4} \mathrm{PF}_{6}$ was added dropwise, followed by $\mathrm{H}_{2} \mathrm{O}(50 \mathrm{~mL})$. The aqueous phase was extracted with $\mathrm{CH}_{2} \mathrm{Cl}_{2} / \mathrm{MeNO}_{2}(100 \mathrm{~mL}, 5: 1)$, and the organic phase washed with $\mathrm{H}_{2} \mathrm{O}(3 \times 100 \mathrm{~mL})$, dried $\left(\mathrm{Na}_{2} \mathrm{SO}_{4}\right)$, and evaporated to yield $\left[\mathbf{1 2 H}_{3}\right]\left[\mathrm{PF}_{6}\right]_{3}(0.8 \mathrm{~g}, 79 \%)$ as a white solid. $\mathrm{Mp}$ 
142-145 ${ }^{\circ} \mathrm{C} ;{ }^{1} \mathrm{H}$ NMR $\left(500 \mathrm{MHz}, \mathrm{CD}_{3} \mathrm{CN}\right) \delta 4.31(\mathrm{~s}, 6 \mathrm{H}), 4.33(\mathrm{~s}, 6 \mathrm{H}), 4.71(\mathrm{~s}, 6 \mathrm{H})$, $7.48-7.56(\mathrm{~m}, 12 \mathrm{H}), 7.61(\mathrm{~d}, J=8 \mathrm{~Hz}, 6 \mathrm{H}), 7.92(\mathrm{~d}, J=8 \mathrm{~Hz}, 6 \mathrm{H}), 7.97(\mathrm{~s}, 3 \mathrm{H}) ;{ }^{13} \mathrm{C}$ NMR $\left(125 \mathrm{MHz}, \mathrm{CD}_{3} \mathrm{CN}\right) \delta 45.3,50.9,51.0,125.2,127.8,129.2,130.0,130.5,130.6$, $130.7,139.4,141.3,141.4$; HRMALDI-MS $\mathrm{m} / z$ calcd for $\mathrm{C}_{51} \mathrm{H}_{49} \mathrm{~N}_{3} \mathrm{Cl}_{3}, 808.2992$ $\left[M-\mathrm{PF}_{6}-2 \mathrm{HPF}_{6}\right]^{+}$, found 808.3099.

$\left[\mathbf{4 H}_{3}\right]\left[\mathbf{P F}_{\mathbf{6}}\right]_{\mathbf{6}} \cdot\left[\mathbf{1 2 H}_{3}\right]\left[\mathrm{PF}_{6}\right]_{3}(0.5 \mathrm{~g}, 0.4 \mathrm{mmol})$ dissolved in DMF $(10 \mathrm{~mL})$ was added dropwise over $2 \mathrm{~h}$ to a solution of 4,4'-bipyridine (1.6 g, $10.0 \mathrm{mmol})$ in anhydrous DMF (43 mL) under an argon atmosphere at $100{ }^{\circ} \mathrm{C}$. The reaction mixture was then left to stir at $100{ }^{\circ} \mathrm{C}$ for an additional $70 \mathrm{~h}$. The solvent was removed under vacuum, and the remaining solid was washed with hexanes: $\mathrm{CH}_{2} \mathrm{Cl}_{2}(5 \times 100 \mathrm{~mL}, 1: 1)$ and then dissolved in a small volume of $\mathrm{MeOH}(10 \mathrm{~mL})$. A saturated aqueous solution of $\mathrm{NH}_{4} \mathrm{PF}_{6}$ was added dropwise, followed by $\mathrm{H}_{2} \mathrm{O}(100 \mathrm{~mL})$. The aqueous phase was extracted with $\mathrm{CH}_{2} \mathrm{Cl}_{2}: \mathrm{MeNO}_{2}(70 \mathrm{~mL}, 1: 1)$, and the organic phase washed with $\mathrm{H}_{2} \mathrm{O}(4 \mathrm{x} 100 \mathrm{~mL})$, dried $\left(\mathrm{Na}_{2} \mathrm{SO}_{4}\right)$, and evaporated to yield $\left[4 \mathrm{H}_{3}\right]\left[\mathrm{PF}_{6}\right]_{6}$ as a yellow solid $(0.6 \mathrm{~g}, 67 \%)$. Mp $250{ }^{\circ} \mathrm{C}$ (decomposition); ${ }^{1} \mathrm{H}$ NMR (500 MHz, $\left.\mathrm{CD}_{3} \mathrm{CN}\right) \delta 4.32(\mathrm{~s}, 6 \mathrm{H}), 4.33(\mathrm{~s}, 6 \mathrm{H}), 5.78$ $(\mathrm{s}, 6 \mathrm{H}), 7.54(\mathrm{~d}, J=8.5 \mathrm{~Hz}, 6 \mathrm{H}), 7.56-7.63(\mathrm{~m}, 12 \mathrm{H}), 7.75-7.78\left(\mathrm{dd}, J^{l}=1.5 \mathrm{~Hz} J^{2}=4.5\right.$ $\mathrm{Hz}, 6 \mathrm{H}), 7.89$ (d, $J=8.5 \mathrm{~Hz}, 6 \mathrm{H}), 7.95(\mathrm{~s}, 3 \mathrm{H}), 8.32$ (d, $J=7.0 \mathrm{~Hz}, 6 \mathrm{H}), 8.80-8.87$ (m, $12 \mathrm{H}) ;{ }^{13} \mathrm{C}$ NMR $\left(125 \mathrm{MHz}, \mathrm{CD}_{3} \mathrm{CN}\right) \delta 50.8,51.1,63.4,121.7,125.2,126.2,127.7,129.5$, $129.9,130.7,131.1,132.1,134.3,140.9,141.3,141.4,144.9,151.0,154.7 ;$ FAB-MS $m / z$ $[M]^{+} 2043.5$.

$\left[\mathbf{6 H}_{3}\right]\left[\mathbf{P F}_{\mathbf{6}}\right]_{9} \cdot 3,5$-Di-tert-butyl benzylbromide $(0.16 \mathrm{~g}, 0.60 \mathrm{mmol})$ was added to $\left[4 \mathrm{H}_{3}\right]\left[\mathrm{PF}_{6}\right]_{6}(0.05 \mathrm{~g}, 0.02 \mathrm{mmol})$ in a $\mathrm{CHCl}_{3} / \mathrm{MeCN}$ solution $(3.5 \mathrm{~mL}, 4: 3)$ under an argon atmosphere. The temperature was raised to $75{ }^{\circ} \mathrm{C}$ and the reaction mixture subsequently 
stirred for an additional $48 \mathrm{~h}$. The solvent was evaporated off and the product purified by column chromatography $\left(\mathrm{SiO}_{2}, \mathrm{MeOH}: 2 \mathrm{M} \mathrm{NH}_{4} \mathrm{Cl}_{\mathrm{aq}}: \mathrm{MeNO}_{2}, 7: 2: 1\right)$. The excess of $\mathrm{MeNO}_{2}$ and $\mathrm{MeOH}$ were then removed under vacuum such that the product still remained dissolved and a saturated aqueous solution of $\mathrm{NH}_{4} \mathrm{PF}_{6}$ was added dropwise. The aqueous phase was extracted with $\mathrm{CH}_{2} \mathrm{Cl}_{2}: \mathrm{MeNO}_{2}(50 \mathrm{~mL}, 1: 3)$, and the organic phase washed with $\mathrm{H}_{2} \mathrm{O}(3 \times 100 \mathrm{~mL})$, dried $\left(\mathrm{Na}_{2} \mathrm{SO}_{4}\right)$, and evaporated to yield $\left[6 \mathrm{H}_{3}\right]\left[\mathrm{PF}_{6}\right]_{9}$ as a yellow solid (0.75 g, $74 \%)$. Mp 198-200 ${ }^{\circ} \mathrm{C} ;{ }^{1} \mathrm{H}$ NMR $\left(500 \mathrm{MHz}, \mathrm{CD}_{3} \mathrm{CN}\right) \delta 1.32(\mathrm{~s}, 54 \mathrm{H})$, 4.26-4.32 (br, 12H), $5.76(\mathrm{~s}, 6 \mathrm{H}), 5.86(\mathrm{~s}, 6 \mathrm{H}), 7.41(\mathrm{~d}, J=1 \mathrm{~Hz}, 6 \mathrm{H}), 7.56(\mathrm{~d}, J=8 \mathrm{~Hz}$, $6 \mathrm{H}), 7.59(\mathrm{t}, J=1 \mathrm{~Hz}, 3 \mathrm{H}), 7.53-7.65(\mathrm{~m}, 12 \mathrm{H}), 7.92(\mathrm{~d}, J=8 \mathrm{~Hz}, 6 \mathrm{H}), 7.97(\mathrm{~s}, 3 \mathrm{H}), 8.36$ (d, $J=7 \mathrm{~Hz}, 6 \mathrm{H}), 8.39(\mathrm{~d}, J=7 \mathrm{~Hz}, 6 \mathrm{H}), 8.96(\mathrm{~d}, J=7 \mathrm{~Hz}, 6 \mathrm{H}), 8.99(\mathrm{~d}, J=7 \mathrm{~Hz}, 6 \mathrm{H})$; ${ }^{13} \mathrm{C}$ NMR $\left(125 \mathrm{MHz}, \mathrm{CD}_{3} \mathrm{CN}\right) \delta 30.3,34.6,50.9,51.2,64.0,65.3,123.7,124.0,124.9$, 127.4, 127.6, 127.7, 129.6, 130.6, 131.0, 132.3, 134.4, 141.3, 141.4, 145.6, 145.9, 150.2, 150.6, 152.3 (two aromatic signals are overlapping); FAB-MS $m / z[M]^{+} 3087.7$.

13. A suspension of 2,3-dihydroxynaphthalene (6.3 g, $39 \mathrm{mmol}), 2-[2-(2-p-$ tolylsulfonyloxy)ethoxy]ethanol $(30.0 \mathrm{~g}, 99 \mathrm{mmol}), \mathrm{K}_{2} \mathrm{CO}_{3}(16.3 \mathrm{~g}, 118 \mathrm{mmol})$ in dry $\mathrm{MeCN}(100 \mathrm{~mL})$ under argon was heated under reflux for $24 \mathrm{~h}$. After the reaction mixture had cooled down to room temperature, it was filtered and the residue was washed with $\mathrm{MeCN}(100 \mathrm{~mL})$. The filtrate and the washings were combined and solvent removed under vacuum. The resulting residue was subjected to column chromatography $\left(\mathrm{SiO}_{2}\right.$, hexanes:Me $\mathrm{CO}, 1: 1$ to $\left.1: 2\right)$ to afford $\mathbf{1 3}$ as a yellow oil $(11.7 \mathrm{~g}, 70 \%) .{ }^{1} \mathrm{H}$ NMR $\left(500 \mathrm{MHz}, \mathrm{CDCl}_{3}\right) \delta 3.5-3.6(\mathrm{~m}, 4 \mathrm{H}), 3.62-3.70(\mathrm{~m}, 8 \mathrm{H}), 3.72-3.78(\mathrm{~m}, 4 \mathrm{H}), 3.90(\mathrm{t}, J=$ $5 \mathrm{~Hz}, 4 \mathrm{H}), 4.22(\mathrm{t}, J=5 \mathrm{~Hz}, 4 \mathrm{H}), 7.10(\mathrm{~s}, 2 \mathrm{H}), 7.27-7.29(\mathrm{~m}, 2 \mathrm{H}), 7.61-7.62(\mathrm{~m}, 2 \mathrm{H}) ;{ }^{13} \mathrm{C}$ 
NMR (125 MHz, $\left.\mathrm{CDCl}_{3}\right) \delta 61.5,68.2,69.4,70.2,70.8,72.6,108.3,124.1,126.2,129.2$, 148.7; HRMS(EI) $m / z$ calcd for $\mathrm{C}_{22} \mathrm{H}_{32} \mathrm{O}_{8}, 424.2097[M]^{+}$, found 424.2101.

14. 2,3-Bis(2-\{2-[2-(2-hydroxy)ethoxy]ethoxy\}ethoxy)naphthalene (13) (11.5 g, 27 $\mathrm{mmol}), \mathrm{NEt}_{3}(27.3 \mathrm{~g}, 270 \mathrm{mmol})$ and a few crystals of DMAP were dissolved in dry $\mathrm{CH}_{2} \mathrm{Cl}_{2}(100 \mathrm{~mL})$. A solution of tosyl chloride $(22.1 \mathrm{~g}, 120 \mathrm{mmol})$ in dry $\mathrm{CH}_{2} \mathrm{Cl}_{2}(250$ $\mathrm{mL}$ ) was added dropwise during $2 \mathrm{~h}$ to this solution. The reaction mixture was stirred overnight and then carefully added to $5 \mathrm{M} \mathrm{HCl}(50 \mathrm{~mL})$ and ice. The organic phase was then washed with $2 \mathrm{M} \mathrm{HCl}(100 \mathrm{~mL})$, and brine $(100 \mathrm{~mL})$. This solution was dried $\left(\mathrm{MgSO}_{4}\right)$ and the solvents were removed under vacuum to afford a brown oil. This oil was subjected to column chromatography $\left(\mathrm{SiO}_{2}\right.$, hexanes: $\mathrm{Me}_{2} \mathrm{CO}, 5: 2$ to $\left.3: 2\right)$ to afford $\mathbf{1 4}$ as a pale yellow oil (15 g, $75 \%)$. ${ }^{1} \mathrm{H}$ NMR $\left(500 \mathrm{MHz}, \mathrm{CDCl}_{3}\right) \delta 2.39(\mathrm{~s}, 6 \mathrm{H}), 3.59-3.64$ (m, 4H), 3.67-3.73 (m, 8H), 3.87-3.94 (m, 4H), $4.14(\mathrm{t}, J=5 \mathrm{~Hz}, 4 \mathrm{H}), 4.24(\mathrm{t}, J=5 \mathrm{~Hz}$, 4H), $7.14(\mathrm{~s}, 2 \mathrm{H}), 7.29(\mathrm{~d}, J=8 \mathrm{~Hz}, 4 \mathrm{H}), 7.28-7.32(\mathrm{~m}, 2 \mathrm{H}), 7.63-7.67(\mathrm{~m}, 2 \mathrm{H}), 7.77$ (d, $J=8.0 \mathrm{~Hz}, 4 \mathrm{H}) ;{ }^{13} \mathrm{C} \mathrm{NMR}\left(125 \mathrm{MHz}, \mathrm{CDCl}_{3}\right) \delta 21.5,68.3,68.6,69.2,69.5,70.7,70.8$, 108.4, 124.1, 126.2, 127.8, 129.2, 129.7, 132.8, 144.7, 148.9; HREI-MS $m / z$ calcd for $\mathrm{C}_{36} \mathrm{H}_{44} \mathrm{O}_{12} \mathrm{~S}_{2}, 732.2274[M]^{+}$, found 732.2281.

Tris-crown ether 7. A solution of 2,3,6,7,10,11-hexahydroxytriphenylene (2.2 g, 6.7 mmol) (HHTP) and 1,2-bis(2-\{2-[2-(2-p-tolylsulfonyloxy)ethoxy]ethoxy\}ethoxy) benzene (14) (14.8 g, $20 \mathrm{mmol})$ in anhydrous DMF (500 mL) was added dropwise during 24 $\mathrm{h}$ to a suspension of cesium carbonate $(26.7 \mathrm{~g}, 80 \mathrm{mmol})$ in anhydrous DMF $(300 \mathrm{~mL})$, while stirring vigorously at $100{ }^{\circ} \mathrm{C}$ under argon. The suspension was stirred at $100{ }^{\circ} \mathrm{C}$ for 5d. Upon cooling down to room temperature, the suspension was filtered through a celite 
pad. The residue was washed with $\mathrm{CHCl}_{3}(500 \mathrm{~mL})$. The combined organic solvents were concentrated under vacuum and the resulting material subjected to column chromatography $\left(\mathrm{SiO}_{2}, i-\mathrm{PrOH}: \mathrm{CHCl}_{3}, 3: 97\right.$ to $\left.10: 90\right)$ to afford the solid that was washed with EtOAc to yield 7 as an off-white solid (0.3 g, $5 \%$ ). Mp 142-143 ${ }^{\circ} \mathrm{C} ;{ }^{1} \mathrm{H}$ NMR (500 $\left.\mathrm{MHz}, \mathrm{CDCl}_{3}\right) \delta 3.85-3.95(\mathrm{br}, 24 \mathrm{H}), 3.96-4.09(\mathrm{~m}, 24 \mathrm{H}), 4.20-4.26(\mathrm{~m}, 12 \mathrm{H}), 4.33-4.40$ (m, 12H), 7.04 (s, 6H), 7.25-7.27 (m, 6H); 7.58-7.59 (m, 6H), $7.73(\mathrm{~s}, 6 \mathrm{H}) ;{ }^{13} \mathrm{C}$ NMR $\left(125 \mathrm{MHz}, \mathrm{CDCl}_{3}\right) \delta 68.9,69.6,69.7,69.9,71.3,71.4,107.1,107.7,123.6,124.0,126.1$, 129.1, 148.5, 148.8; HRMALDI-MS $m / z$ calcd for $\mathrm{C}_{84} \mathrm{H}_{96} \mathrm{O}_{24} \mathrm{Na}, 1511.6189[M+\mathrm{Na}]^{+}$, found 1511.6160.

$\left[\mathbf{5 H}_{3}\right]\left[\mathbf{P F}_{\mathbf{6}}\right]_{9} \cdot 3,5$-Di-tert-butyl benzylbromide $(0.16 \mathrm{~g}, 0.60 \mathrm{mmol})$ was added to a $\mathrm{CHCl}_{3} / \mathrm{MeCN}(3 \mathrm{~mL}, 2: 1)$ solution of $\left[4 \mathrm{H}_{3}\right]\left[\mathrm{PF}_{6}\right]_{6}(0.05 \mathrm{~g}, 0.02 \mathrm{mmol})$ and the tris-crown ether $2(0.08 \mathrm{~g}, 0.06 \mathrm{mmol})$ under an argon atmosphere. The tempera-ture was then raised to $75^{\circ} \mathrm{C}$, and the reaction mixture subsequently stirred for an additional $48 \mathrm{~h}$. The solvent was evaporated off and the crude product purified by column chromatography $\left(\mathrm{SiO}_{2}, \mathrm{MeOH}: 2 \mathrm{M} \mathrm{NH}_{4} \mathrm{Cl}_{\mathrm{aq}}: \mathrm{MeNO}_{2}, 7: 2: 1\right)$. The excess of $\mathrm{MeNO}_{2}$ and $\mathrm{MeOH}$ was then removed under vacuum, such that the product still remained dissolved and a saturated aqueous solution of $\mathrm{NH}_{4} \mathrm{PF}_{6}$ was added dropwise. The aqueous phase was extracted with $\mathrm{CH}_{2} \mathrm{Cl}_{2}: \mathrm{MeNO}_{2}(50 \mathrm{~mL}, 1: 3)$, and the organic phase washed with $\mathrm{H}_{2} \mathrm{O}(3 \times 100 \mathrm{~mL})$, dried $\left(\mathrm{Na}_{2} \mathrm{SO}_{4}\right)$, and evaporated to yield $\left[5 \mathrm{H}_{3}\right]\left[\mathrm{PF}_{6}\right]_{9}$ as a dark greenish solid $(0.03 \mathrm{~g}, 33$ \%). ${ }^{1} \mathrm{H}$ NMR (600 MHz, $\left.\mathrm{CD}_{3} \mathrm{CN}\right) \delta 1.31(\mathrm{~s}, 54 \mathrm{H}), 3.63-4.32(\mathrm{~m}, 66 \mathrm{H}), 4.55-4.62(\mathrm{~m}$, 6H), 4.78-4.89 (m, 6H), 4.91-4.98 (m, 6H), $5.74(\mathrm{~s}, 6 \mathrm{H}), 5.77(\mathrm{~s}, 6 \mathrm{H}), 6.78-6.90(\mathrm{~m}$, 6H), 6.78-6.90 (m, 6H), 7.38 (s, 6H), $7.39(\mathrm{~d}, J=1 \mathrm{~Hz}, 6 \mathrm{H}), 7.44$ (s, 3H), 7.46 (d, $J=8$ $\mathrm{Hz}, 6 \mathrm{H}), 7.57(\mathrm{t}, J=1 \mathrm{~Hz}, 3 \mathrm{H}), 7.56-7.0(\mathrm{~m}, 12 \mathrm{H}), 7.68(\mathrm{~d}, J=8.5 \mathrm{~Hz}, 6 \mathrm{H}), 8.36(\mathrm{~d}, J=$ 
$7 \mathrm{~Hz}, 6 \mathrm{H}), 8.39(\mathrm{t}, J=7 \mathrm{~Hz}, 6 \mathrm{H}), 8.91(\mathrm{~d}, J=7 \mathrm{~Hz}, 6 \mathrm{H}), 8.97(\mathrm{~d}, J=7 \mathrm{~Hz}, 6 \mathrm{H}) ;{ }^{13} \mathrm{C}$ NMR (125 MHz, $\left.\mathrm{CD}_{3} \mathrm{CN}\right) \delta 30.3,34.6,50.9,51.0,63.9,65.3,67.3,67.4,70.4,70.4$, $70.5,71.7,104.5,112.2,121.0,121.1,122.7,123.7,124.2,125.8,127.3,127.4,129.5$, $129.7,129.9,130.2,131.7,133.2,134.7,137.3,138.2,145.2,145.5,146.2,147.6,149.9$, 150.4, 152.4; HRESI-MS $m / z$ calcd for $\mathrm{C}_{198} \mathrm{H}_{234} \mathrm{~N}_{9} \mathrm{O}_{24} \mathrm{P}_{6} \mathrm{~F}_{36}, 1330.5067$ [M-3PF $\mathrm{PF}_{6}$, found 1330.5019.

$\left[\mathbf{8 H}_{3}\right]\left[\mathbf{P F}_{\mathbf{6}}\right]_{9}$. The tris-crown ether $\mathbf{7}(0.09 \mathrm{~g}, 0.06 \mathrm{mmol})$ and $\left[\mathbf{4 H}_{3}\right]\left[\mathrm{PF}_{6}\right]_{9}(0.05 \mathrm{~g}, 0.02$ mmol) were dissolved in $\mathrm{CHCl}_{3}: \mathrm{MeCN}(3.5 \mathrm{~mL}, 3: 2)$ and di-tert-butyl benzylbromide $(0.17 \mathrm{~g}, 0.60 \mathrm{mmol})$ was then added to the reaction mixture that was subsequently heated to $75{ }^{\circ} \mathrm{C}$ under an argon atmosphere for $48 \mathrm{~h}$. The solution was cooled down to room temperature, the solvent evaporated under vacuum, and the residue purified by column chromatography $\left(\mathrm{SiO}_{2}, \mathrm{MeOH}: 2 \mathrm{M} \mathrm{NH}_{4} \mathrm{Cl}_{\mathrm{aq}}: \mathrm{MeNO}_{2}, 7: 2: 1\right)$. The excess of $\mathrm{MeNO}_{2}$ and $\mathrm{MeOH}$ were then removed under vacuum such that the product still remained dissolved and a saturated aqueous solution of $\mathrm{NH}_{4} \mathrm{PF}_{6}$ was added dropwise. The aqueous phase was extracted with $\mathrm{CH}_{2} \mathrm{Cl}_{2} / \mathrm{MeNO}_{2}(50 \mathrm{~mL}, 1: 5)$, and the organic phase washed with $\mathrm{H}_{2} \mathrm{O}$ (4 x $100 \mathrm{~mL})$, dried $\left(\mathrm{Na}_{2} \mathrm{SO}_{4}\right)$, and evaporated to yield $\left[\mathbf{8 H}_{3}\right]\left[\mathrm{PF}_{6}\right]_{9}$ as a brownish solid $(0.025$ g, $23 \%) .{ }^{1} \mathrm{H}$ NMR $\left(600 \mathrm{MHz}, \mathrm{CD}_{3} \mathrm{CN}\right) \delta 1.31(\mathrm{~s}, 54 \mathrm{H}), 3.68-4.32(\mathrm{~m}, 60 \mathrm{H}), 4.38-4.44$ (m, 6H), 4.53-4.60 (m, 6H), 4.80-4.86 (m, 6H), 4.93-4.97 (m, 6H), $5.61(\mathrm{~s}, 6 \mathrm{H}), 5.74(\mathrm{~s}$, 6H), 7.21 (s, 6H), 7.28-7.33 (m, 6H), $7.35(\mathrm{~s}, 3 \mathrm{H}), 7.37-7.43(\mathrm{~m}, 12 \mathrm{H}), 7.56-7.58(\mathrm{br}$, 3H), 7.58-7.61 (m, 12H), 7.66-7.71 (m, 12H), $8.30(\mathrm{~d}, J=7 \mathrm{~Hz}, 6 \mathrm{H}), 8.32(\mathrm{~d}, J=7 \mathrm{~Hz}$, $6 \mathrm{H}), 8.78(\mathrm{~d}, J=7 \mathrm{~Hz}, 6 \mathrm{H}), 8.97(\mathrm{~d}, J=7 \mathrm{~Hz}, 6 \mathrm{H}) ;{ }^{13} \mathrm{C} \mathrm{NMR}\left(125 \mathrm{MHz}, \mathrm{CD}_{3} \mathrm{CN}\right) \delta$ $30.3,34.6,50.9,51.0,63.8,65.3,67.3,67.7,70.3,70.4,70.6,71.8,104.5,107.4,121.0$, $122.7,123.7,124.2,124.3,125.7,126.1,127.2,127.3,129.0,129.4,129.7,129.8,130.3$, 
$131.7,133.2,134.5,137.2,138.1,145.2,145.4,146.2,147.9,149.9,150.3,152.4$

HRESI-MS $m / z$ calcd for $\mathrm{C}_{210} \mathrm{H}_{240} \mathrm{~N}_{9} \mathrm{O}_{24} \mathrm{P}_{6} \mathrm{~F}_{36}{ }^{3+} 1380.5224\left[M-3 \mathrm{PF}_{6}\right]^{3+}$, found 1380.5228 .

Absorption and Luminescence Spectra. The absorption and luminescence spectra were recorded with a Perkin Elmer $\lambda 40$ spectrophotometer and a LS 50 spectrofluorimeter, respectively, on air equilibrated acetonitrile (Merck Uvasol ${ }^{\mathrm{TM}}$ ) solutions at room temperature, with concentrations ranging from $4 \times 10^{-6}$ to $1 \times 10^{-4} \mathrm{~mol}$ $\mathrm{L}^{-1}$. Solutions were examined in 1-cm spectrofluorimetric and 4-cm spectrophotometric quartz cells. The experimental error on the wavelength values was estimated to be \pm 1 nm.

Electrochemical Measurements. Electrochemical experiments were carried out by employing cyclic voltammetry (CV) and differential pulse voltammetry (DPV) techniques on argon-purged acetonitrile (Romil HiDry ${ }^{\mathrm{TM}}$ ) solutions at room temperature, with an Autolab 30 multipurpose instrument interfaced to a PC. The working electrode was a glassy carbon electrode (Amel; $0.07 \mathrm{~cm}^{2}$ ); its surface was routinely polished with a $0.3 \mu \mathrm{m}$ alumina-water slurry on a felt surface, immediately prior to use. The counter electrode was a Pt wire, separated from the solution by a frit; an Ag wire was employed as a quasi-reference electrode, and ferrocene was present as an internal standard. The concentration of the compounds examined was $4 \times 10^{-4} \mathrm{~mol} \mathrm{~L}^{-1}$; tetraetylammonium hexafluorophosphate (TEAP) $0.04 \mathrm{~mol} \mathrm{~L}^{-1}$ was added as supporting electrolyte. Cyclic voltammograms were obtained at sweep rates varying from $20 \mathrm{mV} \mathrm{s}^{-1}$ to $1 \mathrm{~V} \mathrm{~s}^{-1}$; differential pulse voltammograms were recorded at sweep rates of 20 and $4 \mathrm{mV} \mathrm{s}^{-1}$ with a pulse height of 75 or $10 \mathrm{mV}$, respectively, and a duration of $40 \mathrm{~ms}$. The potential window examined was from -2 to $+2 \mathrm{~V}$ versus SCE. The experimental error on the 
potential values was estimated to be $10 \mathrm{mV}$. The diffusion coefficient and the number of exchanged electrons were determined by chronoamperometric experiments with the method reported by Bard et al. ${ }^{\mathrm{S} 3}$ In such experiments, a Pt disk ultramicroelectrode (25 $\mu \mathrm{m}$ radius) was employed as the working electrode.

Molecular Modeling. Molecular modeling was carried out both by inspection of a physical CPK space-filling molecular model and molecular mechanics calculations. The latter were performed by employing the MM3 force field as implemented in the Tinker 4.0 package (Copyright 1990-2003 Dr. J.W. Ponder, Biochemistry and Molecular Biophysics, Washington University School of Medicine, St. Louis, MO). The MM3 calculations were carried out on the molecular elevator $\left[5 \mathrm{H}_{3}\right]^{9+}$. The model of $\left[5 \mathrm{H}_{3}\right]^{9+}$ was constructed from the crystal structure of its parent ${ }^{\mathrm{Sla}}$ supramolecular bundle, $\left[2 \cdot 3 \mathrm{H}_{3}\right]^{3+}$, by adding $4,4^{\prime}$-bipyridinium and 3,5-di-tert-butyl groups to each leg of the $\left[3 \mathrm{H}_{3}\right]^{3+}$ component. Such a structure was then energy-minimized until a gradient lower than $0.1 \mathrm{kcal} / \mathrm{mol} / \mathrm{A}$ had been reached. The models of the various deprotonated forms of the molecular elevator were obtained from that of $\left[5 \mathrm{H}_{3}\right]^{9+}$ after removal of the proton(s) from the ammonium unit(s) and manual displacement of the corresponding crown ether loop(s) to surround the bipyridinium unit(s). The structures so obtained were then energy-minimized.

\section{References}

(S1) (a) Balzani, V.; León, C. M; Credi, A.; Lowe, J. N.; Badjic, J. D.; Stoddart, J. F.; Williams, D. J. Chem. Eur. J. 2003, 9, 5348-5360. (b) Badjic, J. D.; Balzani, V.; Credi, A.; Lowe, J. N.; Silvi, S.; Stoddart, J. F. Chem. Eur. J. 2004, 10, 1926-1935. 
(S2) (a) Beattie, D. R.; Hindmarsh, P.; Goodby, J. W.; Haslam, S. D.; Richardson, R. M. J. Mater. Chem. 1992, 2, 1261-1288. (b) Boden, N.; Borner, R. C.; Bushby, R. J.; Cammidge, A. N.; Jesudason, M. V. Liq. Cryst. 1993, 15, 851-858. (c) Fyfe, M. C. T.; Lowe, J. N.; Stoddart, J. F.; Willians, D. J. Org. Lett. 2000, 2, 1221-1224.

(S3) Denuault, G; Mirkin, M. V.; Bard, A. J. J. Electroanal. Chem. 1991, 308, 27-38.

\section{References in Full from the Text}

(15) (c) Jeppesen, J. O.; Nielsen, K. A.; Perkins, J.; Vignon, S. A.; Di Fabio, A.; Ballardini, R.; Gandolfi, M. T.; Venturi, M.; Balzani, V.; Becher, J.; Stoddart, J. F. Chem. Eur. J. 2003, 9, 2982-3007.

(16) (f) Liu, Y.; Flood, A. H.; Bonvallet, P.; Vignon, S. A. ; Northrop, B. H.; Tseng, H.R.; Jeppesen, J. O.; Huang, T. J.; Brough, B.; Baller, M.; Magonov, S.; Solares, S. D.; Goddard III, W. A.; Ho, C.-M.; Stoddart, J. F. J. Am. Chem. Soc. 2005, 127, 9745-9759.

(22) (b) Ashton, P. R.; Ballardi R.; Balzani, V.; Baxter, I.; Credi, A.; Fyfe, M. C. T.; Gandolfi, M. T.; Gómez-López, M.; Martínez-Díaz, M. V.; Piersanti, A.; Spencer, N.; Stoddart, J. F.; Venturi, M.; White, A. J. P.; Williams, D. J. J. Am. Chem. Soc. 1998, 120, 11932-11942.

(26) (b) Ashton, P. R.; Ballardini, R.; Balzani, V.; Constable, E. C.; Credi, A.; Kocian, O.; Langford, S. J.; Preece, J. A.; Prodi, L.; Schofield, E. R.; Spencer, N.; Stoddart, J. F.; Wenger, S. Chem. Eur. J. 1998, 12, 2413-2422. 